\title{
Normativa educativa y su impacto en los actores involucrados en la formación escolar.
}

\section{Educational regulations and their impact on the actors involved in school training.}

Normativa educativa y su impacto en la formación escolar.

Gary Osman Moreira Moreira ${ }^{(\mathbf{1})}$

Nacira Viviana Alvarado Chilán ${ }^{(2)}$

Rosa Patricia Peñafiel Lino ${ }^{(3)}$

Beatriz Edith Moreira Peñafiel. ${ }^{(4)}$

(1) Escuela de Educación Básica Fiscal “24 de Mayo" N². Portoviejo- Manabí- Ecuador. e-mail: gonaries71@hotmail.com

ORCID: https://orcid.org/0000-0002-6622-9137

(2) Escuela de Educación Básica Fiscal “24 de Mayo” N². Portoviejo, Manabí, Ecuador. e-mail: viviana_alvarado1973@hotmail.com

ORCID: https://orcid.org/0000-0001-9890-6019

(3) Escuela de Educación Básica Fiscal "24 de Mayo” N 2. Portoviejo, Manabí, Ecuador. e-mail: rosapatriciapl79@hotmail.com

ORCID: https://orcid.org/0000-0002-8877-8743

(4) Escuela de Educación Básica Fiscal "24 de Mayo” № 2. Portoviejo, Manabí, Ecuador. e-mail: edithpenafiel19@gmail.com

ORCID: https://orcid.org/0000-0001-6704-7100

Contacto: gonaries71@ @otmail.com

\section{Resumen}

La presente investigación se realizó con docentes y representantes legales de la ciudad de Portoviejo-Manabí-Ecuador. El objetivo del trabajo, fue identificar el impacto que causola 
normativa en la educación y cuya problemáticase enfocó en métodos que reúnan todas las necesidades y expectativase influya a su vez en la motivación de cognición de la comunidad educativa. La investigación se desarrolló con una metodología empírica, basada en las experiencias de los participantes, cuyos mecanismos fueron organizados bajo la planificación de eventos investigativos de carácter técnico científico, con la finalidad de validar las variables de estudio como eran la normativa educativa y su impacto en la formación escolar, a través de la observación. Para esto se manejó una encuesta como instrumento para la recolección de datos, la cual fue aplicada a representantes legales y maestros en la que se evidencio,el desconocimiento sobre la normativa. Esta aplicación tuvo a su vez un enfoque descriptivo, observacional, cualitativo y cuantitativo, que permitió alcanzar los objetivos inmersos. La intención de avalar y desarrollar los derechos y obligaciones constitucionales en el ámbito pedagógico permitió la expedición de la Ley Orgánica de Educación Intercultural del Ecuador, la normativa educativa vigente. Los resultados obtenidos en la investigación, permitieron la elaboración y ejecución de una propuesta de concientización, basadas en las carencias expuestas por los actores de la comunidad educativa que consistió en comprometerlos, mediante talleres participativos, que ayuden a incrementar sus conocimientos sobre el marco legal, alcanzando los objetivos propuestos en la normativa.

Palabras clave: Regulación educativa, capacitación escolar, maestros, representantes legales

\section{Abstract}

This research was conducted with teachers and legal representatives of the city of PortoviejoManabí-Ecuador. The main objective was to identify the impact that the regulations caused in education and whose problems were focused on methods that meet all the needs and expectations and in turn influence the motivation of cognition in the educational community. In the process of this work, an empirical methodology was developed, based on the experiences of the participants, whose mechanisms were organized under the planning of research events of a technical-scientific nature, in order to validate the study variables such as educational regulations and its impact on school education, through observation. For this, a survey was handled as an instrument for data collection, which was applied to legal representatives and teachers in which it was evidenced, ignorance about the regulations. This application in turn had a descriptive, observational, qualitative and quantitative approach, which allowed to achieve the immersed objectives. The intention of endorsing and developing constitutional rights and obligations in the pedagogical field allowed the issuance of the Organic Law of Intercultural Education of Ecuador, the current educational regulations. The results obtained in the research, allowed the elaboration and execution of an 
awareness proposal, based on the deficiencies exposed by the actors of the educational community that consisted of engaging them, through participatory workshops, that help increase their knowledge about the legal framework, achieving the objectives proposed in the Regulations.

Keywords: Educational regulation, school training, LOEI, teachers, legal representatives

\section{Introducción}

En el siglo XXI la calidad es una necesidad en todos los ámbitos y el Ecuador no está exento de ese requerimiento. Y a la que se compromete como lo manifiesta en su(Constitución de la República del Ecuador, 2008)Sección Quinta, art. 26; "La educación es un derecho de las personas a lo largo de su vida y un deber ineludible e inexcusable del Estado. Constituye un área prioritaria de la política pública y de la inversión estatal, garantía de la igualdad e inclusión social y condición indispensable para el buen vivir. Las personas, las familias y la sociedad tienen el derecho y la responsabilidad de participar en el proceso educativo". Por lo que el ser humano es normado e incluso limitado para evitar la posibilidad de un desfase con relación a sus compañeros.

Castagnasso, (2019), asevera que la educación es el proceso general que envuelve a la sociedad y al hombre. Es la preocupación de todas las colectividades desde que la supervivencia de la vida social, su continuidad, estabilidad y progreso dependen fundamentalmente de ella, sobre la base del comentario de Castagnasso sabemos que la instrucción es fundamental para el desarrollo y logro de un país, que busca a través de la preparaciónacadémica de sus mandantes, el progreso igualitario e inclusivo que permita una sociedadecuánime, donde todos tengan oportunidades y no que el impedimento al acceso de esta sea su limitante.

Al respecto Maya (2019), expone que "el liderazgo directivo influye directamente en la consecución de una educación de calidad, es decir, que uno de los factores que hacen posible la denominada educación de calidad, es la presencia de un liderazgo directivo efectivo, asertivo, democrático, participativo, que involucre al personal docente en los planes de trabajo de modo activo y protagónico, cuando este estilo de liderazgo se hace presente, la organización camina hacia la calidad ...”.Por tal motivo aquellos países con mayor desarrollo cuentan con una formación envidiable, porque han llevado más allá de la normativa el proceso educativo, permitiéndoles la organización necesaria para encaminarse a un desarrollo constructivista, adecuándolo a su día a día por medio de un aprendizaje activo. 
Es así como es posible remontarse a lo más relevante, el aprendizaje. Y para este requerimiento el paísdebiócrear una normativa que reúna las condiciones necesarias para incorporar encada actor y nivel de educación; habilidades, destrezas, conocimientos, conductas o valores, las mismas que a través delasexperiencias (ventajas y desventajas), y la observación, se convirtieron en el tema de análisis de esta investigación. En este sentido Villón (2019), expresa que, "las cualidades de la estructura curricular responden a la existencia de elementos organizacionales que permiten evaluar el alcance, secuencia y pertinencia de los aprendizajes...",

Sobre la base del comentario de Tomalá,referente a las estructuras en educación inicial, considero valido de resaltar dos de los cinco enfoques que se le da a las teorías y fundamentos en el texto,en donde en primera instancia, se hace un reconocimiento del niño como un ser único e irrepetible,seguido a esto se realizala respectiva vinculación yparticipación de la familia en el proceso educativo, los cuales son parte importante en nuestro trabajo por ser además de los docentes, ellos actores principales y activos en el proceso de educación, permitiéndoles concebir de manera cercana la realidad en cuanto al aprendizaje se trata.

Por otra parte la UNESCO (2015), afirma enlos resultados del Foro Mundial sobre Educación, que "la capacidad de aprendizaje a lo largo de toda la vida dentro de una educación de calidad es un tema priorizado que necesita un cambio de modelo que nos aleje de las ideas de enseñanza y capacitación y se aproxime a las de aprendizajes, de una instrucción transmisora de conocimientos a un aprendizaje para el desarrollo personal, y de una adquisición de competencias especiales a un descubrimiento de más amplio espectro y la liberación y el dominio del potencial creador. Este cambio es necesario en todos los niveles y en todas las modalidades de la educación..." (Ruiz, 2018)

Con relación a lo expresado por Ruiz, coincido que los países en el mundo están convencidos de la importancia en el modelo de educación y por tal motivo se encuentran buscando nuevas metodologíaspara ser implementadas en sus países,tal es el caso del Ecuador, que enlos últimos nueve años se encuentra regido por una nueva normativa, la misma que lo ha llevado a fomentar e implementar nuevas estrategias de aprendizaje, para permitirles a los actores implícitosen las competencias socio educativas, desenvolverse de manera óptima y eficaz, y para afirmar esta premisa exponemos el caso de los docentes con títulos ajenos a la licenciatura de educación y que gozando de un cargo definitivo en el Ministerio de Educación, tienen que optar por una maestría referente a la docencia dando muestra de compromiso a lo expresado en la normativa. 
Otro ejemplo serialas realizadas en las escuelas a través de las TIC( Tecnologías de la Información y la Comunicación), cuya técnica en pleno siglo XXI a nivel escolar, es considerada como la innovación educativa del momento, la cual permite acceder de forma oportuna a los docentes y al alumnado a información privilegiada, obteniendo de esta forma cambios determinantes de enseñanza -aprendizaje en el quehacer diario del aula, otra comparación digna de mencionar, esla oferta de becasacadémicas a estudiantes universitarios que les permite acceder a estudios en diferentes países del mundo poniéndolos de igual a igual con los retos del mundo.

"La importancia de las políticas educativas se ha resaltado a nivel internacional desde hace varios años, comprendiéndose su rol para la reforma y mejoramiento del sistema educativo" (Araujo, 2015), en el Ecuador sin duda alguna con las reformas que se hicieron en la Ley, se aposto por ella, como mediopara salir del subdesarrollo educativocultural y económico, además de cumplir con el mandato constitucional del'Buen Vivir', Los estudios alrededor de este marco legal representan una inyección de conocimiento desde el ámbito académico, que sirve como base para el futuro diseño de políticas públicas educativas (Morán, 2017).

Esta necesidad dapaso a la reforma de la normativa vigente,que con la intención de garantizar, desarrollar y profundizar los derechos y obligaciones constitucionales en el ámbito educativo, seexpide la LOEI (Ley Orgánica de Educación Intercultural del Ecuador), la cual fue publicada en el segundo suplemento del Registro Oficial 417 del 31 de marzo de 2011 y la que esobjeto de estudio en nuestra investigación.

En el presente artículo se pone énfasis en los resultados que la normativa tenga hacia la validez e invalidez de los mismos, ya que de esto dependerá la permanencia de la misma en las sucesiones de gobiernos que pudiesen ocurrir, volviéndose por su eficacia en una política de estado sustentable en el tiempo.

Como referencia una de las necesidades que recoge la normativa vigente, son los requerimientos que tenían los maestros anteriormente ,concerniente a su remuneración, lo cual los obligaba a distraer sus obligaciones, en busca de satisfacer las necesidades básicas de subsistencia y otra comolas requeridas por personas con necesidades especiales, las cuales no podían asistir a la institución educativa de su conveniencia violentando con esta limitación sus derechos,esta condición queda salvada con lo expresado en la,(Constitución de la República del Ecuador, 2008), expresada en el art. 2, literal e, referente a la inclusión: "La atención e integración prioritaria y especializada de las niñas, niños y adolescentes con discapacidad o que padezcan enfermedades 
catastróficas de alta complejidad" logrando a través de este artículo presente en la ley, hacer realidad un derecho que les permite desarrollar todas sus potencialidades.

Siendo el problema que motivó esta investigación, la efectividad de la normativa además de comprobar quereúna todas las necesidades educativas e influya directamente en la formación de conciencia de la comunidad.

El presente artículo tiene por objetivoidentificar el impacto que causo la normativa en la educación y cuya problemática se enfocó en métodos que reúnan todas las necesidades y expectativas e influya a su vez en la motivación de cognición de la comunidad educativa.

\section{Material y Métodos}

En el desarrollo del presente trabajo de investigación, se utilizó una metodología empírica, basada en las experiencias de los participantes, cuyos mecanismos fueron organizados bajo la planificación de eventos investigativos de carácter técnico científico, con la finalidad de validar las variables de estudio como eran la normativa educativa y su impacto en la formación escolar, a través de la observación.

Se utilizó como instrumento de evaluación, una encuesta para la recolección de datos, la misma que fue aplicada a representantes legales y maestros, en la que se evidencio la existencia de varias apreciaciones sobre la normativa, entendiéndose la disconformidad y avenencia en la aplicación de la misma.

La investigación se desarrolló bajo un enfoquedescriptivo, observacional, cualitativo y cuantitativo, que permitió alcanzar los objetivos inmersos, que permitió alcanzar el cumplimiento de los objetivos, dichos trabajos se realizaron en el cantón Portoviejo-Manabí-Ecuador en donde se tomó contacto a través de la web con los encuestados utilizando la aplicación del método descriptivo, ya que detalla las particularidades del problema investigado, definiendo a su vez, las variables de estudio como son la normativa educativa y su impacto en la formación escolar.

Se trabajó también con el método analítico porque se pudieron analizarlos datos arrojados en la encuesta, que a su vez fueron tabulados e interpretados con la ayuda de la técnica del cuestionario, describiendo así la problemática planteada y finalmente también se utilizó el método sintético al realizar las conclusiones de dicha investigación. 
El tipo de la muestra que se utilizó para esta investigación fue aleatoria simple, la cual se tomó del grupo de padres de familia, y docentes que participaron. Se emplearon materiales tecnológicos como: calculadora, laptop o computadora, el programa de Word, el programa Excel para la tabulación de resultados.

Este involucramiento de los actores coadyuvo a la calidad dela investigación, alcanzando con ello la recopilación veraz entre las diferentes opiniones de los actores encuestados, para poder así comprobar la problemática planteada. Se prefirió excluir a estudiantes para evitar vulnerar sus derechos; según, consta en el capítulo tercero de la(Ley Organica de Educación Intercultural, 2011), art. 7, literal 1. El margen de error en esta investigación consistió en el 0,05\%de los resultados arrojados mediante la técnica de encuesta.

\section{Resultados}

En la realización de esta investigación se aplicaron encuestas dirigidas a maestros y representantes legales de estudiantes de instituciones educativas particulares y fiscales del cantón PortoviejoManabí-Ecuador, cuyo instrumento de investigación tuvo 19 ítems, basados en las variables de estudio, las cuales fueron direccionadas con el propósito de interpretar en los actores sus habilidades, destrezas, conocimientos, conductas o valores en la implementación de la Ley en su cotidianidad educativa, y de esta forma lograr establecerel impacto que está causando la normativa en la educación.Para demostrar y tabular los porcentajes de nuestra investigación utilizamos dos tablas que detallaremos a continuación, tabla 1.

\begin{tabular}{|l|l|l|}
\hline Actores involucrados & Número & Porcentaje \\
\hline Maestros & 41 & $56.00 \%$ \\
\hline Representantes legales & 32 & $44.00 \%$ \\
\hline Total de encuestados & 73 & $100 \%$ \\
\hline
\end{tabular}

Tabla 1 Actores involucrados en el ámbito escolar

La tabla 1 muestra el grupo y la cantidad de encuestados que intervinieron en esta investigación, cuyo objetivo principal fue identificar el impacto que causo la normativa en la educación, adicional se tiene a la problemática, la cual se enfocó en métodos que reúnan todas las necesidades y expectativas que influya a su vez en la motivación de cognición de la comunidad educativa. Por otro lado, también seanalizó la permisibilidad de la normativa educativa hacia la comunidad,y para poder lograr el propósito se encuesto un total de 73personas, distribuida de la siguiente manera: 41 maestros y 32 representantes legales de los estudiantes. 
De acuerdo a los resultados obtenidos en las encuestas aplicadas a docentes y representantes legales sobre la normativa educativa y su impacto en la formación escolar, en las diferentes instituciones de la ciudad de Portoviejo, provincia de Manabí, Ecuador, se evidencia que los actores en la comunidad tiene un déficit en el conocimiento de la normativa, esto se manifiesta en los porcentajes obtenidos en la encuesta, obteniendo como resultadosque los dos grupos sumado sus porcentajes de la primer variables no alcanzan el 50\% como para justificar el balance de conocimientosde los entes involucradosy siendo esta variable la más relevante en cuanto a direccionalidad,compromiso y valores que deberían tener los actores inmiscuidos en la investigación.

De la misma manera, es contradictorio observar que los porcentajes reflejados en la segunda variable de $52.24 \%$ en los docentes que tienen poco conocimiento de la normativa para aplicarla en su ámbito laboral. Por otra parte los resultados obtenidos por parte de los padres muestran que este $50.83 \%$ conjuntamente con el obtenido por el grupo anterior, no es un resultado sustancial para el aprovechamiento de los beneficios que tiene la normativa, porque en ambos se refleja el desconocimiento hacia la normativa. Por último el porcentaje de $23.75 \%$ de los docentes y el $28.80 \%$ de los padres de familia de la tercera variable es demasiado elevado tomando en cuenta que ambos grupos encuestados son parte primordial para la consecución de los objetivos expresados en la Constitución de la República del Ecuador y por ende en la normativa actual, tabla 2.

\begin{tabular}{|l|l|l|}
\hline Variables & Docente & $\begin{array}{l}\text { Representantes } \\
\text { legales }\end{array}$ \\
\hline MUCHO & $24.01 \%$ & $20.37 \%$ \\
\hline POCO & $52.24 \%$ & $50.83 \%$ \\
\hline NADA & $23.75 \%$ & $28.80 \%$ \\
\hline TOTAL & $100 \%$ & $100 \%$ \\
\hline
\end{tabular}

Tabla 2. Padres y maestros de instituciones particulares y fiscales del cantón Portoviejo

\section{Discusión}

Por medio de esta investigación se pudo evidenciar, el desconocimiento general de la normativa por parte de los actores en la comunidad educativa llamados a involucrarse en esta tarea tan profunda como es la de educar através de las herramienta otorgadas en la norma donde el gran perdedor termina siendo el educando, con esto me refiero que hay que mejorar los niveles de conocimiento e interpretación de la Norma para ponerla al servicio de la comunidad educativa y aprovechar de esta forma sus bondades que posee la misma. 
Se concuerda con Barraza (2018), en la que señala "La investigación educativa es muy prolífica debido a las diversas necesidades de un mundo en constante cambio. La mayoría de las reformas que pretenden mejorar la calidad de la educación se centran en cambios normativos, que permitan modelar las mejoras esperadas" en base al comentario. Por otra parte, expresa que "el desafío de las organizaciones escolares es interpretar e implementar el conjunto de leyes que constituyen la normativa nacional..." por lo que está en sus manos la aplicación de la misma través de una implementación consciente lograda por medio de perfecta aplicación, para este propósito los involucrados debe tener un conocimiento amplio de los reglamentos que conforman la Ley.

Otros estudios realizados por Flores (2019), hacen alusión a la trasformación de los sistemas educativos del mundo arroja evidencia sobre la importancia que la política educativa posee dentro de las agendas de los gobiernos. Para los interesados en el tema es conveniente describir y discernir los principales elementos de la política pública de educación de los Estados bajo sus respectivos contextos.En base a lo expuesto por Flores coincidimos que el logro sustancial que puede traer consigo una reforma o actualización de la normativa requerirá del compromiso e implementación consciente a través de la destreza con la que se puedan confluir las necesidades de la comunidad educativa y dela intención de la Normativa vigente.

Según Yandún (2015), las normas, principios y la práctica de inclusión educativa no presentan concordancia...", este escrito se refiere a la parte en que la Normativa sufre una distorsión en la aplicación de la misma por parte de los encargados de implementar la directrices de las normas, quedando en ocasiones por fuera el espíritu de la Ley y truncando a la vez el objetivo por la que fue elaborado y perjudicado de una manera tan drástica al ente principal siendo estudiante.

El Tercer Estudio Regional Comparativo y Explicativa (TERCE) tiene entre sus propósitos entregar información relevante que contribuya a la mejora de los sistemas escolares de la región. El TERCE provee a los países de evidencia empírica como un aporte para la elaboración de políticas educativas que hagan efectivo el derecho a una educación de calidad para todas las niñas, niñosde la región (UNESCO, 2015).

En nuestro país el pronunciamiento de la UNESCOque hace a través del estudio realizado por el programa TERCE se encuentra evidenciado en muchos de los contenidos que tienen la normativa y que son de suma importancia para los actores que en un momento han sido relegados o recibían beneficios a medias, tales es el caso de las personas con necesidades educativas especiales y el respeto ala diversidad. 
En esta experiencia se concuerda con Bonet (2019), en la que plantea cada forma de enseñar genera aprendizajes distintos. Es por ello que, estos recursos educativos abiertos abren nuevas posibilidades a la innovación de la estructura educativa tradicional, permitiendo tanto a estudiantes como a docentes disponer de recursos interactivos, dinámicos, flexibles y totalmente libres, capaces de facilitar el acceso a un aprendizaje distinto y de calidad. Ante lo expuesto por Bonet la implementación de las TIC, aparecen como una herramienta alternativa eficaz para la enseñanza y aprendizaje moderno a través de sus técnicas.

Siguiendo lo planteado por Cuasapaz (2019), indica que por décadas la educación pública ha estado llena de falencias, siendo la mayor la falta de cobertura a nivel nacional para todo sector, se supondría que con las reformas realizadas estas falencias se superarían. Sin embargo, se evidencia que lejos de superar las debilidades anteriores en la estructura educativa nacional, las mismas continúan, pues la unificación de la malla educativa es un tema también dejado de lado y esto se evidencia en la diferente educación recibida en escuelas rurales y urbanas en temas de estudio que deberían ser generales por su tipología, lo cual coloca en desventaja a alguno de los grupos sociales inherentes.

Al contrario de lo planteado por el autor Cuasapaz (2019), en nuestro país en la última reforma educativa y la malla curricular los cambios relacionados a la cobertura se vio incrementada significativamente, tanto que es así que en algunas instituciones de orden particular se afectaron hasta el cierre de la misma, debido a que la mayoría de la sociedad opto por la educación pública abierta.

El rol que tiene el profesional del centro le obliga a asumir una responsabilidad que necesariamente tiene que impactar en su propia práctica, pues convertirse en modelo le hace consciente de su rol formador y modelador de la conducta del practicante (Yangin, 2018). Ante lo manifestado vale resaltar lo que está sucediendo con los docentes ya que se encuentran en una constante capacitación aparte de una búsqueda de superación académica como es el caso de los docentes que títulos ajenos a la docencia educativa que están obligados a optar por un mejoramiento académico acorde a su labor y el desafío constante de superación educacional de ellos y de sus educandos.

Se concuerda con Aguilar-Gordón, (2019), en la que afirma que es la educación la que contribuye para el logro de grandes transformaciones de la humanidad y a su vez son las transformaciones educativas respaldadas en la filosofía las que fundamentan, dan sentido y significado a la existencia humana, no sin razón se considera que la educación ha sido históricamente el motor que impulsa 
todo proceso social". Esta premisa es consecuente con la aptitud de la Normativa vigente que dentro de sus objetivos es el de convertirse en el promotor del cambio del país a través de la masificación educativa y el profesionalismo de sus educandos, tal es el caso del impulso que hace el Estado a través de becas a diferentes países del mundo en la profesionalización de los suyos.

\section{Conclusiones}

El desconocimiento proporcional de la normativa por parte de los involucrados en la investigación, terminaron siendo una alerta para la incidencia que puede tener la ley, a favor o en contra de todos los actores de la comunidad educativa y el propósito de la misma dicho pronunciamiento se hace en base a los resultados obtenidos en las encuestas aplicadas a maestros (56\%) y representantes legales (44\%) sobre la normativa educativa y su impacto en la formación escolar.

Además se consideró que el objetivo planteado en la investigación como era el de la incidencia de la norma en la educación,fue másallá de su propósito al lograr establecer una significante deficiencia en común, por parte de los encuestados hacia la normativa como es la de su desconocimiento.

El estudio realizado asevera que en futuras investigaciones se tomaran en cuenta otras variables y objetivos, que permitan ahondar aúnmás en el tema en cuestión para definir estrategias que admitan reducir la brecha de conocimientos de los actores de la educación.Siendo notorio las falencias por parte de docentes y representantes legales de los estudiantes, surgió la necesidad de presentar una propuesta alternativa,que consistirá en comprometer a la comunidad educativa, mediante talleres participativos, para el mejoramiento de su conocimiento e interpretación de la normativa.

\section{Bibliografía}

Aguilar-Gordón, F. D. (2019). Fundamento, evolución, nodos críticos y desafíos de la educación ecuatoriana actual. Revista Actualidades Investigativas en Educación, 32.

Araujo, M. D. (2015). Cambios en la política educativa en Ecuador desde el año 2000. Recuperado de Education for All Global Monitoring Report 2015.Recuperado de http://unesdoc.unesco.org/images/0023/002324/232430s.pdf

Barraza, D. (2018). Gestión de normativa interna en liderazgo educacional. Universidad Finis Terrae (Chile) Facultad de Educación, Psicología y Familia)., 44.

Bonet, M. A. (2019). La utilidad de los REA y su impacto en el aprendizaje de educación superior. Editorial Universitat Politècnica de València. 
Castagnasso, A. C. (2019). Interfaz educativa. In IV Jornadas Estudiantiles de Investigación en Disciplinas Artísticas y Proyectuales. SEDICI, 16.

Cuasapaz, R. J. (2019). La reforma Educativa en el Ecuador, mediante el acuerdo Nro. 020-12, y el impacto en la deserción escolar en la Unidad Educativa Consejo Provincial del Carchi. Quito:UCE, 107.

Ecuador, A. N. (2008). Constitución de la República del Ecuador. Recuperado de https://www.asambleanacional.gob.ec/sites/default/files/documents/old/constitucion_de_bolsi llo.pdf

Flores, J. (2019). La política pública de educación en Colombia y Finlandia: elementos para la construcción de una educación pública en época de Reformas Educativas Globales. Papel Politico.

Maya, E. Z. (2019). Liderazgo Directivo y Educación de Calidad. CIENCIAMATRIA, 16.

Morán Cabrera, K. A. (2017). Marco legal educativo ecuatoriano ¿ Genera posibilidad o limitaciones? Análisis basado en narrativas docentes en torno a la educación inclusiva (Bachelor's thesis. Universidad Casa Grande., 81.

Nacional, A. (2011). Ley Organica de Educación Intercultural.

Ruiz, L. M. (2018). Desafíos de la educación superior. Consideraciones sobre el Ecuador. INNOVA Research Journal, 9.

UNESCO. (2015). Resultados del Foro Mundial sobre la Educación de 2015. Recuperado de https://unesdoc.unesco.org/ark:/48223/pf0000234002_spa?posInSet=1\&queryId=69d3e5c1 a6d3-4b83-876d-62739367c709

Villón Tomalá, A. M. (2019). Análisis del Currículo Ecuatoriano de Educación Inicial 2014. Universidad Nacional de Educación, 9.

Yandún, A. (2015). Inclusión Educativa: Percepciones de los docentes a partir de la aprobación. UDLA , 136.

Yangin Eksi, G. \&. (2018). Exploring the Use of Narratives to Understand Pre-service Teachers'. Australian Journal of Teacher. 